\title{
Effects of Early Life Adverse Experience on Contents of Essential Trace Elements Related to the Antioxidative System in the Adult Mouse Hippocampus
}

\author{
Cho Azuma1', Takeshi Minami², Mayumi Nishi1 ${ }^{*}$ \\ ${ }^{1}$ Department of Anatomy and Cell Biology, Faculty of Medicine, Nara Medical University, Nara, Japan \\ ${ }^{2}$ Laboratory of Environmental Biology, Department of Life Science, Faculty of Science and Technology, Kinki University, \\ Osaka, Japan \\ Email: *nmayumi@naramed-u.ac.jp, aaa@naramed-u.ac.jp
}

How to cite this paper: Azuma, C., Minami, T., \& Nishi, M. (2020). Effects of Early Life Adverse Experience on Contents of Essential Trace Elements Related to the Antioxidative System in the Adult Mouse Hippocampus. Psychology, 11, 314-325.

https://doi.org/10.4236/psych.2020.112020

Received: January 23, 2020

Accepted: February 16, 2020

Published: February 19, 2020

Copyright $\odot 2020$ by author(s) and Scientific Research Publishing Inc. This work is licensed under the Creative Commons Attribution International License (CC BY 4.0).

http://creativecommons.org/licenses/by/4.0/

(c) (i) Open Access

\begin{abstract}
Neonatal maternal separation has been found to affect adult brain function. However, the molecular basis has not been clarified. The purpose of this study was to elucidate the changes in mineral levels related to the antioxidative system in the adult mouse hippocampus after neonatal maternal separation. Male mouse pups in the maternal separation group were separated from their dams for 3 hours daily during the first 2 postnatal weeks. The hippocampal selenium, calcium, zinc, copper, and manganese contents were analyzed with an inductively coupled plasma mass spectrometer at 13 postnatal weeks, and plasma protein carbonyl and 8-OHdG concentrations were examined by enzyme-linked immunosorbent assay. It was found that the hippocampal selenium content was about three times lower in the maternal separation group than that in the control group, representing a significant difference. The hippocampal calcium content was more than 2 times higher in the maternal separation group than that in the control group. The plasma protein carbonyl concentration was about seven-fold higher in the maternal separation group than that in the control group. However, no significant differences were found in the hippocampal calcium, zinc, copper, and manganese contents, or in plasma protein carbonyl and 8-OHdG concentrations between the maternal separation and control groups. Therefore, neonatal maternal separation affects the hippocampal selenium content of adult male mice, but does not affect other mineral contents, such as zinc, copper, manganese, and calcium in the hippocampus.
\end{abstract}




\section{Keywords}

Maternal Separation, Hippocampus, Selenium, Zinc, Protein Carbonyl

\section{Introduction}

During postnatal development, adverse early life experiences affect the formation of neuronal networks and exert long-lasting effects on neural function. Many studies have shown that daily repeated maternal separation (MS) during the neonatal period, one of the most familiar animal models of early life stress, can alter the adult hypothalamic-pituitary-adrenal axis (HPA axis) responsiveness to stress (Lajud et al., 2012; Levine et al., 1991; Levine, 1994) and cause subsequent various kinds of psychological impairments including mood and anxiety disorders during adulthood (Meaney, 2001). Neonatal MS also deteriorates learning and memory. For example, neonatal MS impairs eye-blink classical conditioning (Wilber et al., 2007), special memory as assessed by the Morris water maze test (Huot et al., 2002), novel object recognition in male rats (Aisa et al., 2007), and fear conditioning (Kosten et al., 2006). However, the neural mechanisms underlying how neonatal MS, which is a transient adverse experience during neonatal periods, induces long-lasting effects on learning and memory, remain to be clarified.

Previous studies showed that neonatal MS induces oxidative stress and increases brain lipid peroxidation in rats (Almeida et al., 2018) and also elicits global DNA hypermethylation in the hippocampus (McCoy et al., 2016). Oxidative stress in the brain tissue is induced by the overproduction of reactive oxygen species (ROS) and free radicals, which overwhelms the endogenous antioxidant capacity of the brain (Floyd \& Carney, 1992). Reactive oxygen species and free radicals, such as $\mathrm{O}_{2} \cdot$ (superoxide radical), $\cdot \mathrm{OH}$ (hydroxyl radical), $\mathrm{H}_{2} \mathrm{O}_{2}$ (hydrogen peroxide), and NO (nitric oxide) cause damage to cell components including proteins, lipids, and DNA, which affects cellular signaling (Floyd \& Carney, 1992; Gilgun-Sherki et al., 2001). The brain is more susceptible to oxidative damage than any other part of the body since it uses high levels of oxygen and contains high levels of fatty acids which are more susceptible to peroxidation. Furthermore, brain tissue is rich in iron and excitatory amino acids, and their metabolites produce ROS (Floyd \& Carney, 1992). ROS and free radicals affect glial cells and neurons, inducing neural cell damage.

Endogenous antioxidants such as trace elements reduce the effects of oxidative stress on cells by scavenging the ROS (Rizzo et al., 2010; Hosseinimehr, 2015). For example, selenium (Se) is known as an important component of the antioxidant enzyme, glutathione peroxidase. A previous study showed that selenium deficiency induces a decrease in glutathione peroxidase and glutathione levels, leading to an imbalance of calcium homeostasis in chicken brains (Xu et al., 2013). On the other hand, zinc $(\mathrm{Zn})$, copper $(\mathrm{Cu})$, and manganese $(\mathrm{Mn})$ are essential parts of the antioxidant enzyme, superoxide dismutase (SOD). There- 
fore, deficiencies of selenium, zinc, copper, or manganese can cause oxidative damage to proteins, lipids, and DNA, and cause an imbalance of calcium homeostasis, all of which can induce various kinds of disease. There have been some reports on the correlation between selenium, zinc, and brain function. Ishrat et al. (2009) reported that selenium prevents cognitive decline and oxidative damage in a rat model of streptozotocin-induced experimental dementia of the Alzheimer's type. Another recent study reported that maternal zinc supplementation improves spatial memory in rat pups (Piechal et al., 2012) and selenium deficiency impairs hippocampus-dependent learning (Babür et al., 2019).

However, the effects of neonatal MS on the contents of trace elements in the mouse brain have never been studied. Thus, the purpose of the present study was to investigate the hippocampal contents of selenium, zinc, copper, manganese, and calcium in adult male mice exposed to neonatal MS. Furthermore, we examined plasma concentrations of various oxidative stress markers in adult male mice exposed to neonatal MS. One is protein carbonyl, one of the most harmful oxidative stress markers produced by irreversible oxidative protein modifications. The other is 8-OHdG (8-hydroxy-2'-deoxyguanosine), one of the most common by-products of DNA oxidation.

Here, we showed that neonatal MS significantly decreased the content of selenium in the hippocampus of adult male mice, whereas the contents of zinc, copper, and manganese did not exhibit significant differences between the MS group and the control (Cont) group. Regarding oxidative markers, the plasma concentration of protein carbonyl showed a tendency toward an increase in the MS group, while the plasma concentration of 8-OHdG showed no significant difference between the MS and Cont groups. As far as we know, this is the first paper to investigate the effects of neonatal MS on the contents of trace elements involved in oxidative stress in the hippocampus. The present findings provide an important relationship between psychological stress and trace elements in the hippocampus, which may cause learning and memory impairments.

\section{Materials}

\subsection{Animals}

C57BL/6N female mice at day 13 of pregnancy were purchased from Japan SLC Inc. (Hamamatsu, Japan). They were individually housed and maintained under standard laboratory conditions $\left(23^{\circ} \mathrm{C}, 55 \%\right.$ humidity in a room with a $12-\mathrm{hr}$ light-dark cycle (light on at 8:00 am), food and water ad libitum). The day of the pups' birth was designated as PND 0. All animal protocols were approved by the Animal Care Committee of Nara Medical University and were performed in accordance with the policies established in the NIH Guide for the Care and Use of Laboratory Animals.

\subsection{Chemicals and Reagents}

Protein carbonyl ELISA kit (KPC-250DT, Japan Institute for the Control of Ag- 
ing, Nikken SEIL Co., Ltd., Japan), highly sensitive ELISA kit for 8-hydroxy-2'deoxyguanosine (8-OHdG) (KOG-HS10/E, Japan Institute for the Control of Aging, Nikken SEIL Co., Ltd., Japan), and other chemicals used in the experiment were obtained from commercial sources.

\section{Methods}

\subsection{Maternal Separation}

Pups in the MS group were subjected to daily 3-h MS (9:30 to 12:30) from PND 1 to 14 . Dams were first removed from their home cages and placed in new identical cages until the end of the separation period. All pups were individually placed in a cup on a heating pad maintained at $32^{\circ} \mathrm{C}$. At the end of the separation period, pups were returned to their home cages, followed by reunion with their dams. Pups in the Cont group were left undisturbed with the dam until weaning, except for cage cleaning once a week. All pups were weaned on PND 21 and housed in groups composed of 3 - 4 pups of the same gender.

Mice were sacrificed by decapitation at 13 weeks, and then the hippocampus and blood were collected.

\subsection{Determination of Elements, Plasma Protein Carbonyl, and 8-0HdG Levels}

Male mice at 13 weeks old were sacrificed by decapitation and then the hippocampi were isolated. The hippocampi were dried at $100^{\circ} \mathrm{C}$ for $4 \mathrm{~h}$ and were placed in $2 \mathrm{ml}$ nitric acid at room temperature. After 24 hours, the samples were adjusted to a volume of $3 \mathrm{ml}$ by adding ultrapure water. Selenium, calcium, zinc, copper, and manganese contents of the samples were analyzed by inductively coupled plasma mass spectrometry (ICPM-8500; Shimadzu). Calibration curves were prepared at $0,5,10,20 \mathrm{ppb}$ for selenium, $0,5,10,20 \mathrm{ppb}$ for zinc, 0, 5, 25, $50 \mathrm{ppb}$ for copper, $0,5,25,50 \mathrm{ppb}$ for manganese, and 0, 100, 200, $1000 \mathrm{ppb}$ for calcium. The conditions were $1.2 \mathrm{~kW}$ of power from a radiofrequency generator, a plasma argon flow rate of $1.5 \mathrm{l} / \mathrm{min}$, a cooling gas flow of $7 \mathrm{l} / \mathrm{min}$, a carrier gas flow of $0.61 / \mathrm{min}$, and a sampling depth of $3.5 \mathrm{~mm}$. The element amount was expressed on a dry-weight basis.

Male mice at 13 weeks old were sacrificed by decapitation and blood was collected from the trunk into heparinized tubes. Plasma was obtained by centrifugation and stored at $-80^{\circ} \mathrm{C}$ until analysis. The plasma protein carbonyl concentration was examined by enzyme-linked immunosorbent assay (ELISA) using a protein carbonyl ELISA kit according to the manufacturer's instructions. The plasma 8-OHdG concentration was examined using a highly sensitive ELISA kit for 8-OHdG according to the manufacturer's instructions.

\subsection{Statistical Analysis}

Statistical analyses were performed using GraphPad Prism version 3.0 (GraphPad Software, San Diego, CA, USA). Pearson's correlation was used to investi- 
gate the association between parameters. A two-tailed unpaired Student's t test was used to analyze differences between groups. A $p$ value of less than 0.05 was considered to be significant. Data were expressed as the mean \pm standard deviation.

\section{Results}

\subsection{Selenium, Zinc, Copper, Manganese, and Calcium Contents in the Hippocampi}

Figure 1 shows the average contents of selenium in the hippocampi. The average selenium contents of the hippocampi were $0.104 \pm 0.140 \mu \mathrm{g} / \mathrm{g}$ in the MS group and $0.303 \pm 0.083 \mu \mathrm{g} / \mathrm{g}$ in the Cont group. The hippocampal selenium content was about three times lower in the MS group compared with that in the Cont group. There was a significant difference in the hippocampal selenium content between the MS and Cont groups $(p<0.0001)$. The hippocampal selenium content was significantly reduced due to the stress of neonatal maternal separation.

The average zinc contents of the hippocampi were $39.3 \pm 8.30 \mu \mathrm{g} / \mathrm{g}$ in the MS group and $38.2 \pm 7.18 \mu \mathrm{g} / \mathrm{g}$ in the Cont group (Figure 2). No significant difference was found in the hippocampal zinc content between the MS and Cont groups $(p=0.684)$. The average copper contents of the hippocampi were $16.2 \pm$ $2.08 \mu \mathrm{g} / \mathrm{g}$ in the MS group and $17.4 \pm 2.95 \mu \mathrm{g} / \mathrm{g}$ in the Cont group (Figure 3). Although the MS group showed lower copper content, the difference between the MS and Cont groups was not significant $(p=0.151)$. The average manganese contents of the hippocampi were $1.91 \pm 0.35 \mu \mathrm{g} / \mathrm{g}$ in the MS group and $1.80 \pm$ $0.13 \mu \mathrm{g} / \mathrm{g}$ in the Cont group (Figure 4). No significant difference was found in the hippocampal manganese content between the MS and Cont groups ( $p=$ 0.164). The hippocampal zinc, copper, and manganese contents did not change significantly due to the stress of neonatal maternal separation.

As shown in Figure 5, the average calcium contents of the hippocampi were $3.78 \pm 6.99 \mathrm{mg} / \mathrm{g}$ in the MS group and $1.64 \pm 2.26 \mathrm{mg} / \mathrm{g}$ in the Cont group. The hippocampal calcium content was more than 2 times higher in the MS group than that in the Cont group. However, no significant difference was found in the hippocampal calcium content between the MS and Cont groups $(p=0.423)$.

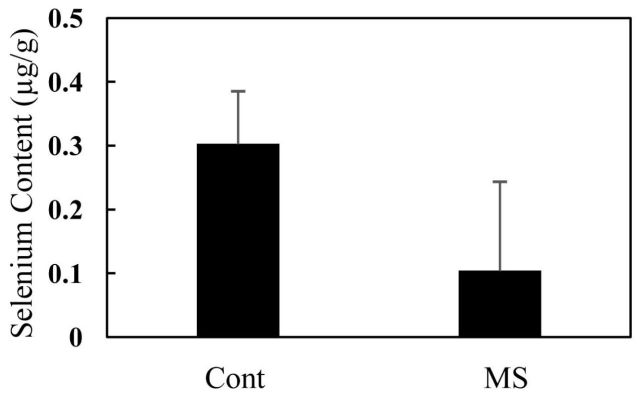

Figure 1. Average selenium contents of the hippocampi in the maternal separation (MS) group and the control (Cont) group. There was a significant difference in the hippocampal selenium content between the MS and Cont groups $(p<0.0001)$. 


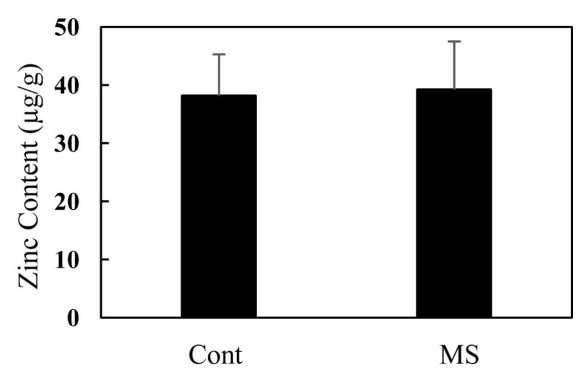

Figure 2. Average zinc contents of the hippocampi in the maternal separation (MS) group and the control (Cont) group. No significant difference was found in the hippocampal zinc content between the MS and Cont groups $(p=0.684)$.

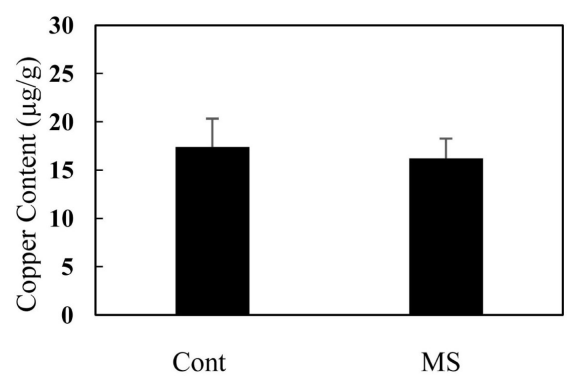

Figure 3. Average copper contents of the hippocampi in the maternal separation (MS) group and the control (Cont) group. There was no significant difference in the hippocampal copper content between the MS and Cont groups $(p=0.151)$.

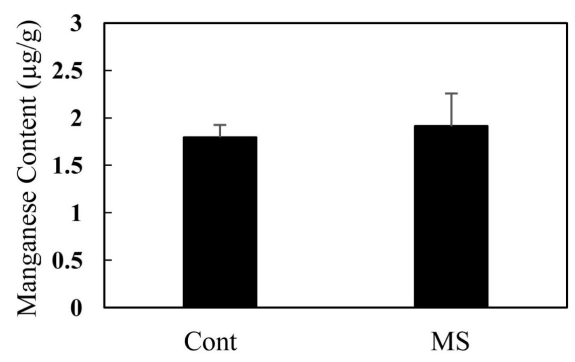

Figure 4. Average manganese contents of the hippocampi in the maternal separation (MS) group and the control (Cont) group. No significant difference was found in the hippocampal manganese content between the MS and Cont groups $(p=0.164)$.

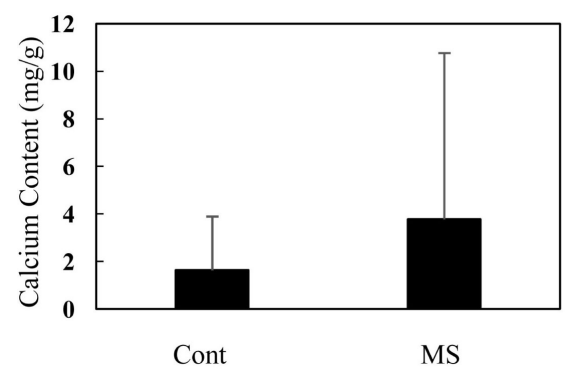

Figure 5. Average calcium contents of the hippocampi in the maternal separation (MS) group and the control (Cont) group. The hippocampal calcium content was more than 2 times higher in the MS group than that in the Cont group. However, no significant difference was found in the hippocampal calcium content between the MS and Cont groups $(p=0.423)$. 


\subsection{Relationships among Element Contents in the Hippocampi}

We analyzed the relationships among hippocampal element contents in the MS group (Table 1). The correlation coefficients were estimated to be 0.583 ( $p=$ $0.006)$ between selenium and zinc contents and $0.585(p=0.007)$ between manganese and copper contents in the MS group. Very significant direct correlations were found between selenium and zinc contents and between manganese and copper contents. In contrast, the correlation coefficient was estimated to be -0.478 ( $p=0.033)$ between selenium and manganese contents in the MS group. A significant inverse correlation was found between selenium and manganese contents. However, no significant correlations were found between zinc and manganese contents, between copper and element contents, such as selenium and zinc, or between calcium and element contents, such as selenium, zinc, manganese and copper in the MS group.

Table 2 lists the relationships among hippocampal element contents in the Cont group. No significant correlations were found among hippocampal element contents, such as selenium, zinc, copper, manganese and calcium, in the Cont group.

\subsection{Concentrations of Protein Carbonyl and 8-0HdG in the Plasma}

The concentrations of protein carbonyl in the plasma were $9.92 \pm 21.7 \mathrm{nmol} / \mathrm{g}$ in the MS group and $1.50 \pm 31.5 \mathrm{nmol} / \mathrm{g}$ in the Cont group (Figure 6). The plasma protein carbonyl concentration was about seven-fold higher in the MS group than that in the Cont group. However, there was no significant difference in the plasma protein carbonyl concentration between the MS and Cont groups ( $p=$ 0.481). As shown in Figure 7, the concentrations of 8-OHdG in the plasma were

Table 1. Relationships among hippocampal element contents in the maternal separation group.

\begin{tabular}{ccccc}
\hline \multirow{2}{*}{ Element } & \multicolumn{4}{c}{ Correlation Coefficient and $p$ Value } \\
\cline { 2 - 5 } & $\mathrm{Zn}$ & $\mathrm{Mn}$ & $\mathrm{Cu}$ & $\mathrm{Ca}$ \\
\hline $\mathrm{Se}$ & $0.583(0.006)$ & $-0.478(0.033)$ & $-0.407(0.075)$ & $-0.201(0.634)$ \\
$\mathrm{Zn}$ & & $-0.144(0.544)$ & $-0.224(0.342)$ & $-0.128(0.762)$ \\
$\mathrm{Mn}$ & & & $0.585(0.007)$ & $0.138(0.744)$ \\
$\mathrm{Cu}$ & & & & $0.141(0.740)$ \\
\hline
\end{tabular}

$p$ Values are indicated in parentheses.

Table 2. Relationships among hippocampal element contents in the control group.

\begin{tabular}{ccccc}
\hline \multirow{2}{*}{ Element } & \multicolumn{4}{c}{ Correlation Coefficient and $p$ Value } \\
\cline { 2 - 5 } & $\mathrm{Zn}$ & $\mathrm{Mn}$ & $\mathrm{Cu}$ & $\mathrm{Ca}$ \\
\hline $\mathrm{Se}$ & $0.183(0.515)$ & $-0.070(0.804)$ & $-0.325(0.238)$ & $-0.277(0.507)$ \\
$\mathrm{Zn}$ & & $-0.294(0.287)$ & $0.130(0.645)$ & $0.498(0.209)$ \\
$\mathrm{Mn}$ & & & $0.251(0.286)$ & $-0.130(0.758)$ \\
$\mathrm{Cu}$ & & & & $0.030(0.943)$ \\
\hline
\end{tabular}

$p$ Values are indicated in parentheses. 


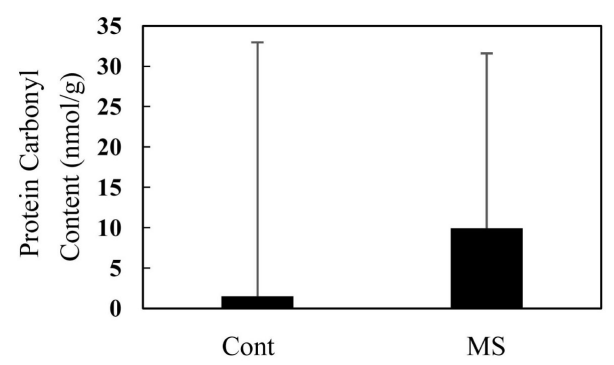

Figure 6. Average protein carbonyl contents of the plasma in the maternal separation (MS) group and the control (Cont) group. Although the plasma protein carbonyl concentration was about seven-fold higher in the MS group compared with that in the Cont group, there was no significant difference in the plasma protein carbonyl concentration between the MS and Cont groups $(p=0.481)$.

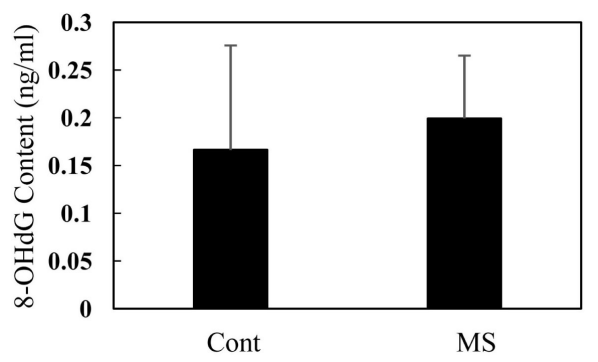

Figure 7. Average 8-OHdG contents of the plasma in the maternal separation (MS) group and the control (Cont) group. There was no significant difference in the plasma 8-OHdG concentration between the MS and Cont groups $(p=0.389)$.

$0.199 \pm 0.066 \mathrm{ng} / \mathrm{ml}$ in the MS group and $0.166 \pm 0.109 \mathrm{ng} / \mathrm{ml}$ in the Cont group. There was no significant difference in the plasma 8-OHdG concentration between the MS and Cont groups ( $p=0.389)$.

In summary of the above results, the stress of neonatal maternal separation significantly reduced hippocampal selenium content, but did not significantly change the hippocampal zinc, copper, manganese, calcium contents, and the plasma protein carbonyl and 8-OHdG concentrations.

\section{Discussion}

Environmental conditions during the neonatal period may affect adult behavioral and neuroendocrine responsiveness, especially affecting programming of the HPA-axis, which influences the cognitive function and pathogenesis of psychiatric disorders. Our previous study indicated that neonatal repeated MS during the first two weeks of life increased basal corticosterone levels in male adult mice (Nishi et al., 2014). Therefore, neonatal MS may cause various kinds of stresses, including oxidative stress in the brain. A previous study showed that increased glucocorticoid levels promoted ROS generation in the hippocampus and cortex (Sapolsky, 1999). Overproduction of ROS and free radicals overrides the endogenous antioxidant capacity of the brain, causes oxidative damage to the brain tissues, and induces lipid peroxidation (Baek et al., 1999; Leutner et al., 2001). Thus, the present study investigated the effects of neonatal MS on the contents 
of trace elements, endogenous antioxidants, that reduce the effect of oxidative stress on cells by scavenging the ROS, and plasma level of oxidative stress markers, protein carbonyl and 8-OHdG.

Selenium, one of the essential trace elements, is known as a key component of the antioxidant enzyme glutathione peroxidase. Therefore, selenium deficiency can reduce the activity of glutathione peroxidase. Huang et al. (2016) reported that selenium deficiency decreased the activities of glutathione peroxidase and catalase in chicken brain. Xu et al. (2013) showed that selenium deficiency decreased glutathione peroxidase and glutathione levels, leading to imbalance of calcium homeostasis in the chicken brain. The present study revealed that the hippocampal selenium content was significantly decreased in the MS group compared with that in the Cont group. Furthermore, the hippocampal calcium content was more than 2-fold higher in the MS group than in the Cont group. These findings suggest that the neonatal MS reduces the hippocampal selenium content and decreases the activity of glutathione peroxidase, which induces an imbalance of calcium homeostasis.

Selenium deficiency was previously associated with a large increase in the protein carbonyl content of the mouse brain (Moskovitz, 2007). Ishrat et al. (2009) reported that a significant decrease in the protein carbonyl and a significant increase in the glutathione peroxidase levels by selenium supplementation were observed in the hippocampus and cerebral cortex. Therefore, it is likely that a decrease in the selenium content can reduce the activity of glutathione peroxidase, resulting in an increase in levels of hydrogen peroxide and hydroxyl radicals that can induce oxidative damage to proteins and increase the protein carbonyl levels. The present study revealed that the hippocampal selenium content was significantly decreased in the MS group compared with that in the Cont group, and the plasma protein carbonyl concentration in the MS group was about 7-fold higher than that in the Cont group. However, there was no significant difference in the plasma protein carbonyl concentration between the MS and Cont groups. Further research is needed to investigate the hippocampal tissue level of protein carbonyl to clarify the correlation between the hippocampal selenium content and the oxidative damage of proteins in the MS group. In contrast, zinc, copper, and manganese are essential components of the antioxidant enzyme, superoxide dismutase. Therefore, zinc, copper, and manganese deficiency can decrease superoxide dismutase activity, which plays an important role in eliminating superoxide. The present study showed that the hippocampal zinc, copper, and manganese contents were not significantly changed in the MS group compared with contents in the Cont group. Therefore, it is likely that the neonatal MS does not affect zinc, copper, and manganese accumulation in the hippocampus.

Regarding the relationships among hippocampal element contents in the MS group, significant direct correlations were found between selenium and zinc contents and between manganese and copper contents, whereas a significant inverse correlation was found between selenium and manganese contents. Namely, 
as selenium level decreases in the hippocampus, zinc level also decreases in the hippocampus, whereas manganese level increases in the hippocampus. However, the selenium content decreased significantly in the hippocampus of the MS group, but the zinc and manganese contents did not change significantly in the hippocampus of the MS group. The difference is interesting and warrants further exploration with increasing numbers of mice.

\section{Conclusion}

The present study indicated that neonatal MS significantly reduced hippocampal selenium content in adult male mice, whereas the contents of zinc, copper, manganese, and calcium were not significantly different between the MS and Cont groups. Furthermore, the plasma concentration of protein carbonyl exhibited a tendency to increase in the MS group compared to that in the Cont group. Taken together, our findings showed that neonatal MS may affect the glutathione peroxidase pathway, but not the superoxide dismutase pathway. Future studies will be required to directly analyze the activities of glutathione peroxidase and superoxide dismutase, and examine oxidative stress markers, protein carbonyl and 8-OHdG, in hippocampal tissues, to investigate more precise mechanisms by which neonatal MS induces oxidative stress. The present study suggests that neonatal MS, a typical animal model of early life adverse experiences, affects the brain by negatively influencing anti-oxidative systems, which induces various kinds of neurodegenerative diseases such as Alzheimer' disease showing cognitive impairments. Our findings would give a possible treatment for individuals suffered from psychological stress in early life to prevent neurodegenerative diseases by regulating contents of trace elements.

\section{Acknowledgements}

This work was supported by Grants-in-Aid for Scientific Research from the Japan Society for the Promotion of Science (15K04189 to C.A.; JP23390040 to M.N.).

\section{Conflicts of Interest}

The authors declare no competing financial or personal interests.

\section{References}

Aisa, B., Tordera, R., Lasheras, B., Del Rio, J., \& Ramirez, M. J. (2007). Cognitive Impairment Associated to HPA Axis Hyperactivity after Maternal Separation in Rats. Psychoneuroendocrinology, 32, 256-266. https://doi.org/10.1016/j.psyneuen.2006.12.013

Almeida, P. M. D., Kamath, S. U., Shenoy, P. R., Bernhardt, L. K., Kishore, A., \& Rai, K. S. (2018). Persistent Attenuation of Brain Oxidative Stress Through Aging in Perinatal Maternal Separated Rat Pups Supplemented with Choline and Docosahexaenoic Acid or Clitoria ternatea Aqueous Root Extract. Folia Neuropathologica, 56, 206-214. https://doi.org/10.5114/fn.2018.78702

Babür, E., Tan, B., Yousef, M., Cinbaş, S., Süer, C., \& Dursun, N. (2019). Deficiency but 
Not Supplementation of Selenium Impairs the Hippocampal Long-Term Potentiation and Hippocampus-Dependent Learning. Biological Trace Element Research, 192, 252-262. https://doi.org/10.1007/s12011-019-01666-X

Baek, B. S., Kwon, H. J., Lee, K. H., Yoo, M. A., Kim, K. W., Ikeno, Y., Yu, B. P., \& Chung, H. Y. (1999). Regional Difference of ROS Generation, Lipid Peroxidation, and Antioxidant Enzyme Activity in Rat Brain and Their Dietary Modulation. Archives of Pharmacal Research, 22, 361-366. https://doi.org/10.1007/BF02979058

Floyd, R. A., \& Carney, J. M. (1992). Free Radical Damage to Protein and DNA: Mechanisms Involved and Relevant Observations on Brain Undergoing Oxidative Stress. Annals of Neurology, 32, S22-S27. https://doi.org/10.1002/ana.410320706

Gilgun-Sherki, Y., Melamed, E., \& Offen, D. (2001). Oxidative Stress Induced Neurodegenerative Diseases: The Need for Antioxidants That Penetrate the Blood Brain Barrier. Neuropharmacology, 40, 959-975. https://doi.org/10.1016/S0028-3908(01)00019-3

Hosseinimehr, S. J. (2015). The Protective Effects of Trace Elements Against Side Effects Induced by Ionizing Radiation. Radiation Oncology Journal, 33, 66-74. https://doi.org/10.3857/roj.2015.33.2.66

Huang, J. Q., Ren, F. Z., Jiang, Y. Y., \& Lei, X. (2016). Characterization of Selenoprotein $\mathrm{M}$ and Its Response to Selenium Deficiency in Chicken Brain. Biological Trace Element Research, 170, 449-458. https://doi.org/10.1007/s12011-015-0486-1

Huot, R. L., Plotsky, P. M., Lenox, R. H., \& McNamara, R. K. (2002). Neonatal Maternal Separation Reduces Hippocampal Mossy Fiber Density in Adult Long Evans Rats. Brain Research, 950, 52-63. https://doi.org/10.1016/S0006-8993(02)02985-2

Ishrat, T., Parveen, K., Khan, M. M., Khuwaja, G., Khan, M. B., Yousuf, S., Ahmad, A., Shrivastav, P., \& Islam, F. (2009). Selenium Prevents Cognitive Decline and Oxidative Damage in Rat Model of Streptozotocin-Induced Experimental Dementia of Alzheimer's Type. Brain Research, 1281, 117-127.

https://doi.org/10.1016/j.brainres.2009.04.010

Kosten, T. A., Lee, H. J., \& Kim, J. J. (2006). Early Life Stress Impairs Fear Conditioning in Adult Male and Female Rats. Brain Research, 1087, 142-150. https://doi.org/10.1016/j.brainres.2006.03.009

Lajud, N., Roque, A., Cajero, M., Gutierrez-Ospina, G., \& Torner, L. (2012). Periodic Maternal Separation Decreases Hippocampal Neurogenesis without Affecting Basal Corticosterone during the Stress Hyporesponsive Period, but Alters HPA Axis and Coping Behavior in Adulthood. Psychoneuroendocrinology, 37, 410-420.

https://doi.org/10.1016/j.psyneuen.2011.07.011

Leutner, S., Eckert, A., \& Müller, W. E. (2001). ROS Generation, Lipid Peroxidation and Antioxidant Enzyme Activities in the Aging Brain. Journal of Neural Transmission (Vienna), 108, 955-967. https://doi.org/10.1007/s007020170015

Levine, S. (1994). The Ontogeny of the Hypothalamic-Pituitary-Adrenal Axis. The Influence of Maternal Factors. Annals of the New York Academy of Sciences, 746, 275-288. https://doi.org/10.1111/j.1749-6632.1994.tb39245.x

Levine, S., Huchton, D. M., Wiener, S. G., \& Rosenfeld, P. (1991). Time Course of the Effect of Maternal Deprivation on the Hypothalamic-Pituitary-Adrenal Axis in the Infant Rat. Developmental Psychobiology, 24, 547-558. https://doi.org/10.1002/dev.420240803

McCoy, C. R., Rana, S., Stringfellow, S. A., Day, J. J., Wyss, J. M., Clinton, S. M., \& Kerman, I. A. (2016). Neonatal Maternal Separation Stress Elicits Lasting DNA Methylation Changes in the Hippocampus of Stress-Reactive Wistar Kyoto Rats. European Journal of Neuroscience, 44, 2829-2845. https://doi.org/10.1111/ejn.13404

Meaney, M. J. (2001). Maternal Care, Gene Expression, and the Transmission of Individ- 
ual Differences in Stress Reactivity Across Generations. Annual Review of Neuroscience, 24, 1161-1192. https://doi.org/10.1146/annurev.neuro.24.1.1161

Moskovitz, J. (2007). Prolonged Selenium-Deficient Diet in MsrA Knockout Mice Causes Enhanced Oxidative Modification to Proteins and Affects the Levels of Antioxidant Enzymes in a Tissue-Specific Manner. Free Radical Research, 41, 162-171. https://doi.org/10.1080/10715760600978823

Nishi, M., Horii-Hayashi, N., \& Sasagawa, T. (2014). Effects of Early Life Adverse Experiences on the Brain: Implications from Maternal Separation Models in Rodents. Frontiers in Neuroscience, 8, 166. https://doi.org/10.3389/fnins.2014.00166

Piechal, A., Blecharz-Klin, K., Pyrzanowska, J., \& Widy-Tyszkiewicz, E. (2012). Maternal Zinc Supplementation Improves Spatial Memory in Rat Pups. Biological Trace Element Research, 147, 299-308. https://doi.org/10.1007/s12011-012-9323-y

Rizzo, A. M., Berselli, P., Zava, S., Montorfano, G., Negroni, M., Corsetto, P., \& Berra, B. (2010). Endogenous Antioxidants and Radical Scavengers. Advances in Experimental Medicine and Biology, 698, 52-67. https://doi.org/10.1007/978-1-4419-7347-4_5

Sapolsky, R. M. (1999). Glucocorticoids, Stress, and Their Adverse Neurological Effects: Relevance to Aging. Experimental Gerontology, 34, 721-732.

https://doi.org/10.1016/S0531-5565(99)00047-9

Wilber, A. A., Southwood, C. J., Sokoloff, G., Steinmetz, J. E., \& Wellman, C. L. (2007). Neonatal Maternal Separation Alters Adult Eyeblink Conditioning and Glucocorticoid Receptor Expression in the Interpositus Nucleus of the Cerebellum. Developmental Neurobiology, 67, 1751-1764. https://doi.org/10.1002/dneu.20549

Xu, S. W., Yao, H. D., Zhang, J., Zhang, Z. W., Wang, J. T., Zhang, J. L., \& Jiang, Z. H. (2013). The Oxidative Damage and Disbalance of Calcium Homeostasis in Brain of Chicken Induced by Selenium Deficiency. Biological Trace Element Research, 151, 225-233. https://doi.org/10.1007/s12011-012-9552-0 